\title{
New LED Luminous Flux Test System and Its Error Analysis
}

\author{
Haoyang Tian ${ }^{1}$, Junhua $\mathrm{Liu}^{2}$, Chenzhao $\mathrm{Fu}^{1}$, Yuanbo $\mathrm{Gu}^{2}$, Qiyu $\mathrm{Lu}^{1}$ and Wei Liao ${ }^{1}$ \\ ${ }^{1}$ State Grid Electric Power Research Institute of Shanghai Electric Power Corporation, Shanghai, 200437, China \\ ${ }^{2}$ Inspection and Maintenance Company, State Grid Shanghai Electric Power Company, Shanghai 200063, China
}

\begin{abstract}
With the continuous improvement of LED luminous efficiency, in the field of general lighting, there is a tendency to replace traditional light sources. But the lack of common standards for LED optoelectronic performance has become a major factor hindering its development. Among them, the luminous flux data is very important not only for determining the LED luminous efficiency and other performance parameters, but also for LED marketing and widely used.
\end{abstract}

Keywords-LED; luminous flux; composite parabolic collector; spatial heterogeneity error; spectral mismatch error

\section{INTRODUCTION}

Due to the potential high luminous efficiency, long life, small size, environmental protection and other characteristics of the LED, it has been developing rapidly in the field of general lighting and has the tendency to replace the traditional light source [1]. Luminous flux data is not only important for determining the luminous efficiency of LEDs, but also in the process of market promotion and replacement of traditional light sources. There is therefore a need for an accurate and easy-to-use luminous flux test method.

Current methods for measuring LED luminous flux can be divided into absolute and relative methods [2]. The absolute method generally uses a distributed photometer to measure the spatial distribution of the luminous intensity of the light source, and then calculate the total luminous flux through software integration. Absolute method is relatively accurate, but the test requires long time, complex equipment, high maintenance costs. The relative method is the method of comparing the measured LED with the standard light source known to the total luminous flux to obtain the total luminous flux. The commonly used instrument is the integrating sphere. This method is simple and fast, but requires the measured lamp and the standard lamp has a similar spectral power distribution. In addition, because the LED is different from the traditional light source, light intensity varies greatly with the angle, different types of LED light distribution is also very different, when the space distribution of measured light is different from standard light, it will When the measured light and standard light space distribution of light is different, will bring space nonuniformity error. And when the integrating sphere is small, self-absorption effect is more serious and the measurement error is bigger. The use of large volume sphere can get relatively accurate results, but the volume is too large to industrial applications.
The new LED luminous flux tester developed in this paper uses the composite parabolic collector to collect the light from the LED light source, and then uses a large area of silicon photodiode to detect the outgoing light. To verify the feasibility of the tester, we use the new tester to measure eight high-power LED which is divided into white and green two groups. Then compared with the luminous flux measured by the photometer, the relative error in the range of $\pm 3.5 \%$. And we use innovative measurement methods to analyze the causes of the error.

\section{New LED LuminOUS FluX TeSTER}

In this paper, we use the composite parabolic concentrator LED luminous flux test method to establish a luminous flux tester CPC-01. The volume of the luminous flux test system is small, the CPC bowl is about $4 \mathrm{~cm}$ in length and about $7 \mathrm{~mm}$ in diameter. And the test is quickly, after the LED is stable, it can read the data immediately [3]. In contrast, the distribution of photometer needs a black chamber in the space. The traditional integrating sphere has a diameter of at least $20 \mathrm{~cm}$, and the smaller the integrating sphere is, the greater the error. In time, the entire test process of the distribution photometer is at least 2 hours.

\section{A. The Principle of CPC for Luminous Flux Test}

$\mathrm{CPC}$ is a non-imaging condenser based on edge optical principles. It is commonly used in solar energy collection. Parabolic optical axis revolves around its own focus at an angle, and then translates and symmetries to get twodimensional CPC. And then rotate around the axis of symmetry to form a three-dimensional CPC [4]. $\phi$ is a variable parameter, $a^{\prime}$ means outlet diameter, $r$ means inlet radius, $\theta_{\max }$ means maximum incidence angle. The contour equation of the column coordinate system is:

$$
\begin{gathered}
r=\frac{2 f \sin \left(\phi-\theta_{\max }\right)}{1-\cos \phi}-a^{\prime} \\
z=\frac{2 f \sin \left(\phi-\theta_{\max }\right)}{1-\cos \phi}
\end{gathered}
$$

Focal length is: 


$$
f=a^{\prime}\left(1+\sin \theta_{\max }\right)
$$

The relationship between the inlet and the outlet radius:

$$
r=\frac{a^{\prime}}{\sin \theta_{\max }} .
$$

The total length $\mathrm{L}$ of $\mathrm{CPC}$ in the z-axis direction is

$$
L=\frac{a^{\prime}\left(1+\sin \theta_{\max }\right) \cos \theta_{\max }}{\sin ^{2} \theta_{\max }}
$$

As long as the angle of incidence of light incident from the entrance point relative to the CPC central axis is less than $\theta_{\max }$, the light can reach the exit after a maximum of one reflection and play the role of convergence light. For light which has angle relative to the central axis less than $\theta_{\max }$ has the highest energy collection rate.

According to the principle of optical reversibility, in this paper we put the LED light source on the exit of CPC, the light emitted by the light source must be reflected and emitted from the entrance. While the maximum angle of the light emitted is $\theta_{\max }$,so CPC can play the role of convergence light. At the same time, almost all of the light can reach the exit after a maximum of one reflection. So, this CPC's light utilization will be much higher than other types of concentrators.

The CPC in this tester uses a CPC bowl with a light angle of $20^{\circ}$, the focal length $\mathrm{f}$ is $4.7 \mathrm{~mm}$, the diameter of the exit $2 \mathrm{a}^{\prime}$ is $7 \mathrm{~mm}$ and the diameter of the entrance $2 \mathrm{r}$ is $20 \mathrm{~mm}$. Add attenuator, cosine correction and $V(\lambda)$ correction between $\mathrm{CPC}$ and silicon photodetector. Using the tracepro simulation Lambertian LED light source for simulation and parameters are set as follows: inner wall coating reflectivity of CPC is $98 \%$, the attenuator has a diameter of $30 \mathrm{~mm}$ and a thickness of $2 \mathrm{~mm}$, the transmittance for all wavelengths is $10 \%$, the absorbance is $90 \%$. The effect of the attenuator is to ensure that the emitted light falls entirely within the detection range of the photovoltaic cell, and reduces the influence of the reflected light of the $V(\lambda)$ correction. The $V(\lambda)$ correction has a diameter of $30 \mathrm{~mm}$, a thickness of $6.5 \mathrm{~mm}$ and a reflectance of 5\%. The cosine correction has a diameter of 30 $\mathrm{mm}$ and a thickness of $2 \mathrm{~mm}$. The bulk scattering performance was described by $\mathrm{ABg} \mathrm{BSDF}$ (bidirectional scattering distribution function) model with a total BTDF of $99 \%$. The detector area is $40 \mathrm{~mm} * 40 \mathrm{~mm}$, the reflectance for all wavelengths are $15 \%$.

For simulation of single light at different angles of incidence, relative error is less than $5 \%$. For simulation of a narrow beam light source with a half-light intensity of $15^{\circ}$, and a Lambertian light source, relative error is less than $2 \%$. The relative error is less than $1.5 \%$ when the LED is off the focal plane center point $\pm 1 \mathrm{~mm}$.

\section{B. The Establishment of the Actual System}

Using different structures of the fixture with different forms of packaging visible light LED, to ensure that the light emitting center of the visible LED is positioned near the bottom of the CPC collector (i.e., the focus plane of the CPC). The CPC fixture combines the radiator. The CPC reflector was used as the luminous flux collection device of the visible light LED, and the silver reflector with high reflectivity was deposited on the inner wall of the reflector cup. Using a large area $(48 * 48 \mathrm{~mm})$ silicon photovoltaic cells as a detector, and install the attenuator, $V(\lambda)$ correction and cosine corrector in turn from the exit port to the light receiving surface of the detector. The output signal of the detector is subjected to current and voltage conversion, and the final display is amplified [3], and then amplified to get the final number. The parameters of the various parts of the actual system are consistent with those in section A.The new luminous flux tester CPC- 01 is a rectangular box with a length of $374 \mathrm{~mm}$, a width of $206 \mathrm{~mm}$ and a height of $70 \mathrm{~mm}$. The range is: $0.01 \mathrm{~lm}$ $\sim 50000 \mathrm{~lm}$.

On the panel, "zero" is the school zero knob, "power" is the power switch. "Gear" is the transfer button which marked with the gear coefficient $\times 1, \times 10, \times 100, \times 1000$. "Luminous flux" is the luminous flux display window, the number need to be multiplied by the corresponding gear coefficient and the calibration factor to get the final luminous flux measurements. "LED sample room" is the placement of LED fixture.

Compare the measured LED luminous flux with the standard LED:

$$
\Phi_{T}=\Phi_{R} \cdot \frac{i_{T}}{i_{R}}
$$

Among them, $\Phi_{T}$ and $\Phi_{R}$ are measured LED and standard LED luminous flux respectively, $i_{T}$ and $i_{R}$ are silicon photovoltaic cell response of measured LED and standard LED respectively.

However, due to process constraints, oxidation and other reasons, CPC wall cannot be a uniform ideal diffuse reflective layer, and the inner wall of the point of the diffuse coefficient is not exactly the same. So, it will introduce the spatial distribution of uneven error when the measured light and standard light space intensity distribution is inconsistent. In addition, the modified detector and the $V(\lambda)$ curve are not exactly the same. So, it will introduce the spectral mismatch error when the measured lamp and standard lamp spectral power distribution is inconsistent. In this paper, the error analysis of the above two aspects is carried out by combining GO-R3000 distribution photometer, integrating sphere and PMS-80 ultraviolet-visible-near infrared spectroscopy system.

\section{EXPERIMENTAL TEST AND ERROR ANALYSIS}

In order to verify the feasibility of the new luminous flux test system, we test 8 high-power LED divided into two groups. 
The first group: No. 1-4, green LED, manufacturer: Cree. The first group of LEDs has similar spectral power distribution and peak wavelengths about 520nm with light for the 100$130{ }^{\circ}$ Lambert body light source.

The second group: No. 5-8, blue and yellow LED, manufacturer: Ningbo Sheng spectrum photoelectric Semiconductor Co., Ltd. The second group of LEDs has similar spectral power distribution but a large difference in light distribution.

We test the various models of LED luminous flux with the new luminous flux tester CPC-01 and GO-R3000 distribution photometer respectively. When using CPC-01 tester, we put the tester host on a flat and solid desktop. After turning on and setting the LED, place the LED in the center of the fixture. Connect the LED and the radiator contact surface to the thermal paste, and then place the LED fixture into the LED sample compartment. Adjust the precision DC power supply to the LED and make the current constant at $350 \mathrm{~mA}$. Adjust CPC01 to the appropriate gear and record the LED operating voltage after 30 mins. Read the number $\mathrm{C}$ from the display window and $\mathrm{C}$ is proportional to the silicon photovoltaic response.

Considering spectral mismatch error and spatial heterogeneity error, we use distribution photometer to test whole luminous flux of LED bare lamp and LED plus CPC reflector bowl respectively. This can separate the spatial

mismatch error and the spectral mismatch error. Using the GO-R3000 distribution photometer (near field, the second detector). During this test, LED uses the same radiator as the CPC-01 test, and the power supply is a precision constant current source to make the current constant at $350 \mathrm{~mA}$. Record the number and voltage and start the distribution of photometer measurement after 30 mins. The entire test process is about 90 minutes. In the test, the vertical angle $\gamma$ is 0 to $180^{\circ}$ at intervals of $0.5^{\circ}$, and the horizontal rotation angle $\mathrm{C}$ is 0 to $355^{\circ}$ at intervals of $5^{\circ}$.

The actual measured luminous flux $\Phi_{C}$ of the new spectrometer is:

$$
\Phi_{C}=K_{C} * C
$$

Among them $K_{C}$ is the optical flux coefficient and $\mathrm{C}$ is the CPC-01 number. Theoretically the coefficient $K_{C}$ should be a fixed value which is equal to $G_{L} / C$. However, the green and white LED have different average value of $G_{L} / C$, two sets of LEDs respectively take different $\mathrm{KC}$ values.

The relative error is:

$$
\Delta_{\mathrm{D}}=\frac{\Phi_{C}-G_{\mathrm{L}}}{G_{\mathrm{L}}}=\frac{K_{C}}{G_{\mathrm{L}} / C}-1
$$

The green and white LED have different average value of $G_{L} / C$, green for 4.9 and white for 5.4. As the two groups of
LEDs have basically the same $G_{L} / G_{C}$, green for 1.24 and white for 1.21. So, the difference between the $G_{L} / C$ of two groups comes mainly from $G_{c} / C$ as known as silicon optical probe spectral mismatch. The luminous flux coefficients for green and white LEDs are 4.9 and 5.4, respectively. The relative error of the new luminous flux meter CPC-01 relative to the distributed photometer is within $3.5 \%$.

\section{A. Spectral mismatch error}

In order to analyze the errors caused by the spectral mismatch of the detector, the relative spectral power distribution of each LED was measured by an integrating sphere with a diameter of $3 \mathrm{~m}$ and a remote PMS-80 UV-Visnear infrared spectroscopy system. The comparison of the relative spectral power distribution of the LED, the distribution photometer detector and the CPC-01 detector sensitivity difference curve is plotted in the same graph. Among them, the sensitivity difference between distributed photometer detector and CPC-01 detector is defined as:

$$
\Delta R=10 \times\left(\frac{\mathrm{R}_{G}}{R_{C}}-1\right)
$$

Among them, $R_{G}$ is the relative spectral response of the distributed photometer probe, and $R_{C}$ is the relative spectral response of the CPC-01 silicon light probe.

$\Delta R$ of green LED is less than 0 near peak wavelength, that is distributed photometer sensitivity is less than CPC probe. So, the $G_{c} / C$ ( the ratio of the luminous flux of LED plus CPC reflector bowl measured by the distribution photometer to the $\mathrm{CPC}$ number) should be small which is measured as 3.938 .

\section{B. Spatial Distribution Uneven Error}

In order to measure the spatial nonuniformity error caused by the internal wall reflectance of CPC, we use tracepro to simulate the ratio $\Phi_{\text {out }} / \Phi_{\text {in }}$ between the luminous flux emitted by the CPC at the time of the CPC and the light source at different reflectances. The parameter setting is the same as section A of chapter2. Using the distributed photometer to measure the spatial light intensity distribution data of 10 different types of LEDs and import it in Tracepro to simulate LED light source. The reflectivity of the inner wall is $70 \%$, $80 \%, 90 \%$ and $100 \%$. Record the ratio $\Phi_{\text {out }} / \Phi_{\text {in }}$ between the luminous flux emitted by 10 CPC entry points and the luminous flux of the light source.

The relative differences of $\Phi_{\text {out }} / \Phi_{\text {in }}$ in the 10 LEDs at different reflectivities, which is normalized to LED number 1. It can be seen that as the CPC wall reflectivity increases, the relative differences of $\Phi_{\text {out }} / \Phi_{\text {in }}$ in the 10 LEDs are reduced. The greater the difference in light distribution, the greater the relative difference caused by the different reflectivity of CPC. Such as No.1 which is similar to Lambertian body and No.6 
which has side light. For narrow beam angle of the LED, CPC wall reflectivity changes have little effect, such as No. 7. When the CPC wall area of different reflectivity, the value of $\Phi_{\text {out }} / \Phi_{\text {in }}$ did not change significantly. The relative difference between the LEDs when the inner wall reflectivity is $100 \%$ is due to the fact that part of the light emitted by the LED does not enter the CPC reflector bowl. In summary, increasing the reflectivity of the $\mathrm{CPC}$ wall can reduce the error caused by the difference in spatial light intensity between the measured lamp and the standard lamp.

\section{DiscUSSION}

New LED luminous flux tester CPC-01 which combinate CPC and silicon photodiode has small size, fast testing, can achieve high accuracy and be used for automated production lines. Through the comparison with the distribution of photometer, the error mainly comes from the spectral mismatch error and spatial distribution of non-uniform two aspects, in which spectral mismatch is the main cause of error. The spectral mismatch error can be eliminated by selecting a standard lamp with similar spectral distribution or combining the spectral power distribution data.

The improvement of the reflectivity of the inner wall can reduce the spatial distribution inhomogeneity error between different distribution LEDs.

In this paper, we do not consider the error caused by the non-neutral reflection of the inner wall coating of CPC. Assume that the silver coating of the CPC wall is the same for the reflectance of each wavelength [6]. LED.

The tester described in this article is only valid for forward

The type and number of LEDs tested in this article are limited. In order to better verify the versatility of the instrument we need to verify for the rest of the monochromatic light (red, blue, yellow, etc.) and different packages, different models of a large number of LED.

\section{CONCLUSION}

This paper describes the use of CPC's new luminous flux tester CPC-01. Four green LEDs with similar spectral power distributions and four white (blue-yellow) LEDs with similar spectral power distributions, which means a total of 8 space with different light of the LED were measured. And then combined with the distribution of photometer on the use of CPC-01 measured data for the error analysis. The flux measurement error mainly comes from two aspects: spectral mismatch error and spatial heterogeneity error. Among them, the spectral mismatch error is the main factor. The relative error between a group of LEDs with similar spectral power distributions is no more than $\pm 3.5 \%$. Increasing the reflectivity of inner wall can effectively reduce the spatial inhomogeneity error.

\section{REFERENCES}

[1] P Hanselaer, A Keppens1, S Forment1, WR Ryckaertl andG Deconinck, "A new integrating sphere design for spectral radiant flux determination of light-emitting diodes," Meas. Sci. Technol. 20095111,2009

[2] CIE 2007 Measurement of LEDs CIE Techinical Report 127-2007, CIE CB, Vienna

[3] Xiaoli Zhou, Shaolong Zhu, Haiping Shen and Muqing Liu,"A new spatial integration method for luminous flux determination of lightemitting diodes," Meas. Sci. Technol , 21(2010)105303(6pp)

[4] Roland Winston, Juan C. Miñano,Pablo Benítez, Nonimaging Optic, Elsevier Academic Press, pp. 56-57

[5] Y. Ohno, A.E. Thompson, "Photmetry-The CIE V $(\lambda)$ Function and What can be Learned from Photometry,"Optical Radiation Hazards, JCNIRP 6/98, CIEx016-998,445-453,1998

[6] Zheng Lv,"Some problems in LED luminous flux testing." China Light \&Lighting, vol.12,2005,pp. 29-30 